\title{
ÜBERSCHUSS VON 17,5 MILLIARDEN EURO
}

Krankenkassen-- Die Finanzreserven der Krankenkassen sind im ersten Halbjahr des Jahres 2017 auf 17,5 Milliarden Euro gestiegen. Die gesetzlichen Krankenkassen erzielten somit in diesem Zeitraum einen Überschuss von rund 1,41 Milliarden Euro. Damit hat sich das Plus des ersten Quartals in Höhe von 612 Millionen Euro mehr als verdoppelt. Die Einnahmen betrugen etwa 116,4 Milliarden Euro und die Ausgaben beliefen sich auf etwa 115 Milliarden Euro. Mit diesem Resultat spricht alles dafür, dass die gesetzlichen Krankenkassen auch im zweiten Halbjahr 2017 ein erhebliches Plus erlangen werden. JG

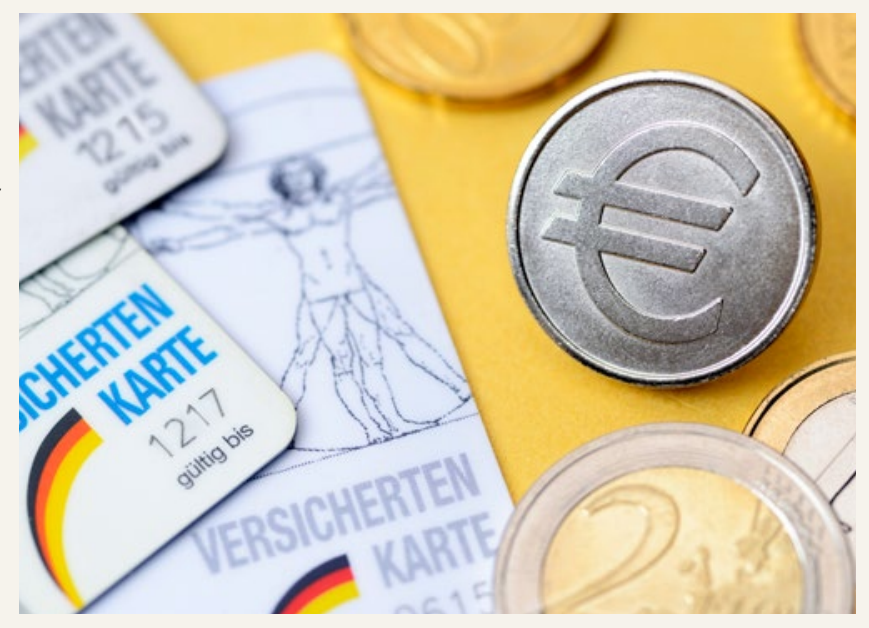

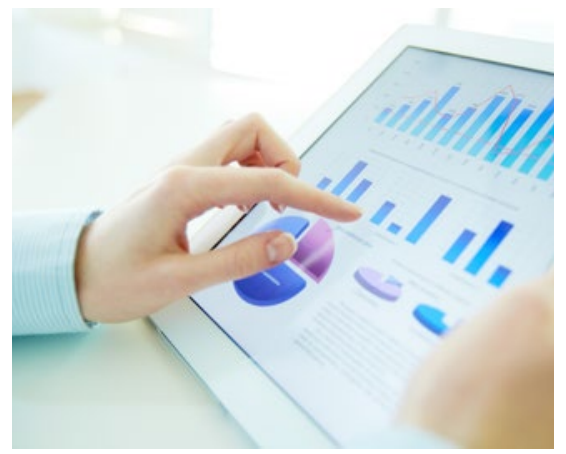

\section{STATISTIK ZEIGT UNTERSCHIEDE}

\section{Einnahme- und Kostenstruktur von Arzt- und}

Zahnarztpraxen-- Nur allzu häufig werden Ärzte und Zahnärzte von der Politik über einen Kamm geschoren. Praxis ist Praxis - alles gleich, so das Credo. Dass es genau so eben nicht ist und die Zahnärzte eher immer "großzügig“ - allerdings nicht immer von Vorteil - in der Gesundheitspolitik mitgedacht werden, ist seit Langem die Einschätzung des Freien Verbandes Deutscher Zahnärzte (FVDZ).

Das jüngste Beispiel dafür, dass sich niedergelassene Zahnärzte von ihren niedergelassenen ärztlichen Kollegen deutlich unterscheiden, stammt vom Statistischen Bundesamt (Destatis), dass sich eingehend mit der Kosten- und Einnah- mestruktur von Arzt- und Zahnarztpraxen beschäftigt hat. Vor allem bei den Einnahmen aus der Abrechnung mit der gesetzlichen Krankenkasse (GKV) zeigten sich erhebliche Unterschiede: Niedergelassene Ärzte erzielen laut der Statistik (Bezugspunkt 2015) 70,4 Prozent ihrer Einnahmen aus der Kassenabrechnung und nur etwas mehr als ein Viertel aus Privatabrechnungen. Bei den Zahnarztpraxen sieht dies ganz anders aus: 48,7 Prozent der Einnahmen werden aus der Kassenabrechnung generiert und gut die Hälfte aus Privatabrechnungen.

Nicht nur hinsichtlich der Einnahmen unterscheiden sich die beiden Berufsgruppen, sondern auch in der Kostenstruktur der Praxis: 507.000 Euro nimmt eine Arztpraxis laut Destatis durchschnittlich jährlich ein, bei einer Zahnarztpraxis sind dies 594.000 Euro. Doch während die Durchschnittarztpraxis „nur“ rund 52 Prozent Kosten hat, liegen diese in der Zahnarztpraxis bei 64 Prozent. Der Reinertrag einer Zahnarztpraxis fällt also trotz höherer Einnahmen deutlich geringer aus als bei einer Arztpraxis. Nun erscheinen diese Zahlen so oder so abenteuerlich hoch, doch die Statistiker haben alle Praxen aller Fachrichtungen einbezogen und dann in Tabellen weiter aufgeschlüsselt. SAS

\section{HOHER VERLUST FÜR ZAHNÄRZTE}

Bürgerversicherung-- Der gesamte Mehrumsatz der privaten Krankenversicherung (PKV) lag 2015 bei 12,63 Milliarden Euro, davon fiel knapp die Hälfte auf ambulante Arztpraxen. Das entspricht im Durchschnitt einem Mehrumsatz von 50.200 Euro pro Praxis. Bei Einführung einer einheitlichen Bürgerversicherung würde dieser Betrag den Praxen fehlen. Bei Zahnarztpraxen würden sogar durchschnittlich 61.900 Euro fehlen, wie ein aktueller Bericht des Wissenschaftlichen Instituts der PKV zeigt.

Deutlich macht die Bedeutung der PKV in der Zahnarztpraxis auch der Anteil der Leistungsausgaben: Die GKV geben 7,9 Prozent für Zahnleistungen aus, bei der PKV sind es 26,8 Prozent - obwohl hier der Anteil der Versicherten nur bei elf Prozent liegt.

MAR 